1 Ministério da Saúde, Secretaria Executiva (SE), Departamento de Monitoramento e Avaliação do SUS (Demas) - Brasília (DF), Brasil. luiz.campelo@saude.gov.br

2 Fundação Oswaldo Cruz (Fiocruz), Escola Nacional de Saúde Pública Sergio Arouca (Ensp), Laboratório de Avaliação de Situações Endêmicas Regionais (Laser) - Rio de Janeiro (RJ), Brasil. Ministério da Saúde, Secretaria Executiva (SE), Departamento de Monitoramento e Avaliação do SUS (Demas) - Brasília (DF), Brasil. Universidade Federal do Rio de Janeiro (UFRJ), Instituto de Estudos em Saúde Coletiva (lesc) - Rio de Janeiro (RJ), Brasil. betuca51@gmail.com

3 Universidade Federal do Pará (UFPA), Instituto de Filosofia e Ciências Humanas, Faculdade de Psicologia e Programa de Pós-Graduação em Psicologia - Belém (PA), Brasil.

pttarso@gmail.com

\section{Análise estratégica do processo de formulação da PM\&A-SUS: lições aprendidas e desafios}

\author{
Strategic analysis of the formulation process of SUS National Policy \\ on Monitoring and Evaluation (PNMEA-SUS): challenges and \\ lessons learned
}

Luiz Marques Campelo', Elizabeth Moreira dos Santos ${ }^{\mathbf{2}}$, Paulo de Tarso Ribeiro de Oliveira ${ }^{\mathbf{3}}$

RESUMO O artigo apresenta a Análise Estratégica (AE) do processo de formulação da Política Nacional de Monitoramento e Avaliação do Sistema Único de Saúde (PNM\&A-SUS). O estudo qualitativo utilizou-se da análise documental e revisão de literatura considerando as três dimensões da AE: pertinência do problema, ou seja, necessidade institucional da política; dos objetivos propostos e das parcerias mobilizadas. A análise mostrou que a formulação vem acontecendo de forma descontínua e não há definição de conceitos, proposições e usos unanimemente aceita entre os stakeholders. O processo interrompido necessita ser retomado para a elaboração consensuada da PNM\&A-SUS, considerando as lições aprendidas e a apropriação realista do contexto.

PALAVRAS-CHAVE Formulação de políticas. Saúde Pública. Avaliação em Saúde.

ABSTRACT This article presents the Strategic Analysis (AE) of the formulation process of SUS National Policy on Monitoring and Evaluation (PNMEA-SUS). The study employed a qualitative document analysis and literature review, accounting for the three dimensions of $A E$ : relevance of the problem (needed institutional policy); proposed objectives and mobilized partnerships. The analysis showed a discontinuous formulation process with relevant controversies regarding evaluation purposes, assumptions, responsibilities and expected competencies among stakeholders. The interrupted formulation process needs to be recommenced, as for the lessons learned and the realistic context assessment.

KEYWORDS Policy making. Public health. Health evaluation. 


\section{Introdução}

O objetivo desse artigo é apresentar os resultados da análise estratégica do processo de formulação da Política de Monitoramento e Avaliação do Sistema Único de Saúde (PM\&A-SUS). O artigo apresenta achados da análise documental relacionada ao processo de formulação a ser completada com a percepção dos atores envolvidos. As reflexões sobre a PM\&A-SUS surgiram a partir da percepção tácita do descompasso entre a formulação e publicação dessa, em comparação com a Política Nacional de Informação e Informática em Saúde (PNIIS).

No curso do processo de institucionalização das práticas de monitoramento, avaliação e disseminação da informação estratégia no âmbito do Sistema Único de Saúde (SUS), a formulação da PM\&A-SUS e a da PNIIS iniciaram-se, pari passu, por meio de processo articulado e inter-relacionado. A premissa comum era a de que, por meio de um processo contínuo e sistemático de Monitoramento e Avaliação (M\&A), seria possível obter informações estratégicas à tomada de decisão, prestação de contas e, consequentemente, contribuir para a transparência e disseminação de informações da gestão do SUS, daí a necessidade de ambas. Entretanto a PNIIS foi publicada em 2015, o que não aconteceu com a PM\&A-SUS.

A formulação de uma política compõe-se de um conjunto de etapas consubstanciadas, não subsumidas, mas interdependentes e que incorpora, em um contexto de consensos e conflitos, aspectos relacionados ao modus operandi escolhido por um Estado para interferir nas relações sociais de seus cidadãos (PAESE; AGUIAR, 2012).

Segundo Teixeira (2002), elaborar uma política significa definir "quem decide o quê, quando, com que consequências e para quem", revelando o posicionamento político-ideológica de um governo:

As políticas públicas traduzem, no seu processo de elaboração e implantação e, sobretudo, em seus resultados, formas de exercício do poder político, envolvendo a distribuição e redistribuição de poder, o papel do conflito social nos processos de decisão, a repartição de custos e benefícios sociais. [...]. (TEIXEIRA, 2002, P. 2).

Nessa ótica, a formulação não é meramente o resultado do conjunto de intenções de um Estado ou de um Governo em um texto normativo que será objeto de apreciação de instâncias superiores. A formulação de uma política é um processo dinâmico, que exige do poder público ações além de uma série de reuniões institucionalizadas. Há de se constituir um processo sistematizado e organizado com a participação da sociedade civil e dos demais atores envolvidos na temática.

Dessa forma instiga-se a necessidade das reflexões apresentadas neste artigo. A análise estratégica realizada considerou as suas três dimensões aplicadas ao processo de formulação: verificação da pertinência do problema, i.e., falta de uma política formalizada; dos objetivos formulados para a política, considerando-se propósitos e usos do monitoramento e avaliação; e caracterização dos atores e parcerias estabelecidas para a sua construção.

\section{O contexto}

O desenvolvimento de marcos referenciais para a avaliação no setor saúde tem sido agenda de gestores nos países membros da Organização para Cooperação e Desenvolvimento Econômico (OCDE) e de organismos internacionais da saúde. Esse processo configura-se como uma elaboração longa e trabalhosa por envolver, em geral, um grande número de parceiros e instituições com interesses diferenciados por vezes convergentes por vezes divergentes em busca de acordos e consensos. Além disso, o ajuste fiscal e a necessidade de rearranjos econômicos e sociais mais equitativos, em especial nos países em desenvolvimento e recém-democratizados, impulsionaram a 
formulação de políticas públicas, com maior destaque àquelas restritivas de gastos.

No contexto da América Latina, a formulação das políticas públicas deu-se concomitantemente ao desenvolvimento de ações de monitoramento e avaliação com vistas ao fortalecimento da accountability e da transparência para a responsabilização dos gastos nas administrações públicas, tendo sido tais ações impulsionadas mais por organismos internacionais que por esforços estatais (VIACAVA ET AL., 2004; SOUZA, 2006; NEIROTTI, 2012).

A análise de políticas públicas é um exercício complexo pela característica multidimensional desse objeto de estudo. Não há, na literatura, definição única, estática e unanimemente aceita de política pública. Também são múltiplas as metodologias e modelos utilizados para descrevê-las. Neste artigo, adota-se a concepção de política pública como um sistema de ações selecionadas por um governo no intuito de se obterem efeitos sobre um ou mais problemas em uma sociedade dinâmica. Embora não consensual quanto às fases estruturais, a literatura pertinente utilizar-se da ideia de ciclo contínuo para descrever as fases das políticas, isto é, a identificação de momentos específicos do processo de construção e implementação de uma política, ressaltando-se a contribuição de tal esquematização para o conhecimento e apropriação da própria política (MATTOS; BAPTISTA, 2015).

A literatura refere-se ao 'ciclo das políticas públicas' como compreendendo diversas etapas ou momentos. Paese e Aguiar (2012) em revisão temática sobre o 'ciclos das políticas púbicas’ considera que caracterização destas fases não é uniforme e muito menos unânime. Em sua revisão, estes autores trazem à tona os achados de Howlett e Ramesh, no final dos anos 1990, que considera as fases de montagem da agenda, formulação da política, tomada de decisão, implementação, monitoramento e avaliação. Incorporam ainda os estudos de Secchi, na primeira década do século XXI, que descreve as etapas como de identificação de problemas, formação de agenda, formulação das alternativas, tomada de decisão, implementação e avaliação. A formulação de uma política, foco das reflexões deste artigo, é a fase em que os governos traduzem em atividades os seus propósitos e plataformas político-ideológicas na busca de efeitos capazes de gerar a mudança, ou seja, transportam seus marcos referenciais, sua intencionalidade, para a prática.

Formular uma política não é uma ação isolada, simples e inicial do 'ciclo das políticas públicas', devendo ser compreendida como um processo que envolve a identificação do cenário do problema que a política em construção pretende enfrentar. Configura-se na fase em que os atores participantes devem reconhecer e definir o problema, correlacionando-o à agenda política. Na formulação, as primeiras decisões são tomadas e, consequentemente, seus efeitos refletirão nas etapas seguintes (SIDNEY, 2007).

Sobre o processo de formulação, Cochran e Malone (1999) apud Sidney (2007) interrogam que, nesse estágio, diversos questionamentos são levantados com o objetivo de se identificar o problema, demostrando a complexidade da formulação. Dentre eles, perguntam qual o problema? Qual o plano ou estratégias para eliminar o problema? Qual são os objetivos e as prioridades? Quais opções são possíveis para se obterem os resultados pretendidos? Quais os custos e benefícios destas opções? Quais externalidades, positivas ou negativas, interferem ou estão associadas com as alternativas possíveis?

Na perspectiva de se considerar a política como um ciclo, o primeiro passo é o reconhecimento do problema abordado como relevante no contexto em que esta se desenvolverá fortemente influenciado por alguns fatores como: os anseios e demandas da sociedade, as necessidades insatisfeitas, julgamento de mérito e valor do problema entre outros (VIANA; BAPTISTA, 2014).

A construção de uma PM\&A-SUS apresenta uma multidimensionalidade de fatores que devem ser objeto de apreciação por 
parte dos formuladores, tais como o normativo jurídico-regulatório vigente, a articulação, aceitabilidade e viabilidade política, os custos e os benefícios dentre outros.

A formulação propriamente dita configura-se na mobilização da ação política e social dos grupos de interesse dotados de fortes recursos e poder durante um governo, e cujos trabalhos resultarão na elaboração de um conjunto de alternativas para o enfrentamento do problema em questão (VIANA; BAPTISTA, 2014). Os custos e possíveis implicações institucionais também devem ser objetos de apreciação no momento da construção do documento normativo (JANNUZZI, 2016).

A literatura tem descrito a formulação como um processo de bastidor, a denominada back-room function, por entender que, apesar de desenvolver-se em meio à estrutura político-burocrata e administrativa de um governo, ela envolve um conjunto menor de atores sob o ponto de vista quantitativo, mas não do ponto de vista do envolvimento institucional. Ou seja, o quantitativo de representantes por instituição é menor, porém o conjunto de instituições partícipes do processo de definição da agenda permanece representado e, em alguns casos, pode ser ampliado. Isso pode ocorrer pelo caráter técnico-especializado exigido aos membros do colegiado de formuladores, que torna a formulação uma fase crítica do 'ciclo das políticas públicas' (SIDNEY, 2007).

Também é valido o pensamento de Vianna (2014) e Jannuzzi (2016) que revela limitações institucionais sofridas pelos grupos de interesse na formulação da política, pois as escolhas, em um governo, estão imersas em uma cultura sócio-política e econômica com aspectos visíveis e outros ocultos.

Em contrapartida, as regras institucionais limitam o raio de ação de quem toma decisões, e a decisão ocorre em instâncias hierárquicas governamentais, dentro de um Estado hierarquizado e que possui formas específicas de funcionamento [...]. (VIANA; BAPTISTA, 2014, P. 67).
Não é a vontade de um técnico do setor público, de um pesquisador acadêmico ou governante eleito, com conhecimento empírico consistente da realidade ou visão ousada, que garante imediatamente a incorporação de uma problemática social na agenda formal de governo [...]. (JANNUZZI, 2016, P. 33).

No Brasil, especificamente no setor saúde, a formulação, implementação e avaliação das políticas públicas têm apresentado, desde a redemocratização do País, em especial pós 2003, forte tendência à governança democrática por meio do fortalecimento à participação da sociedade, transparência, qualidade e eficiência na ação pública (PAESE; AGUIAR, 2012).

Por exemplo, a Política Nacional de Medicamento (PNM), analisada por Machado (2013), é um exemplo dessa nova concepção de Policy Making no País. A PNM contou, em sua formulação, com setores do próprio MS, gestores das três esferas de gestão dos SUS, representantes dos mais variados segmentos do setor público e privado diretamente envolvidos com o objeto da política e com a sociedade, e, indiretamente, por meio do Conselho Nacional de Saúde. Partindo-se da problemática da dificuldade de acesso aos medicamentos, suas causas e consequências foram objeto de debate em diversas arenas decisórias. Machado afirma que foi 'imprescindível a participação popular', independe da metodologia escolhida pelos formuladores é fundamental a abertura e incorporação, em maior ou menor grau, das considerações daqueles que usufruirão ou serão objetos das normativas elencadas na política que há de vir. Paese e Aguiar (2012) reafirmam essa característica da governança democrática no setor saúde brasileiro.

A formulação de uma política de monitoramento e avaliação para o SUS não seria diferente. É fato que as ações de monitoramento e avaliação em saúde ocorrem há décadas no País com uma miscelânea de conceitos, metodologias, instrumentos e abordagens (BRASIL, 2015).

Realizar ações de monitoramento e 
avaliação em um sistema de saúde público e universal não é uma tarefa fácil, em especial no Brasil de 210 milhões de pessoas, tripla carga de doença, necessidades ilimitadas e recursos escassos. Entretanto, torna-se uma ação indispensável à luz da dimensão estratégica do setor e do contexto sócio-político e econômico. Nesse âmbito, as diversas iniciativas de monitoramento e avaliação têm-se utilizado de uma prática mais reflexiva com vistas ao fortalecimento do SUS (SANTOS, 2013).

\section{Política Nacional de Monitora- mento e Avaliação: o labirinto da formulação}

Data da criação do SUS, no início dos anos 1990, a preocupação com a necessidade de monitoramento e avaliação. Em sua legislação estruturante, Lei $\mathrm{n}^{0}$ 8.080/90, o Artigo 15 aborda como competência e atribuições comuns dos entes federados a necessidade de instrumentos e mecanismos de avaliação. No fim dos anos 1990, as preocupações ressurgem no cenário nacional por meio da implantação do Programa Nacional de Avaliação de Serviços Hospitalares (Portaria $\mathrm{GM} \mathrm{n}^{0} 3.408 / 98$ ), do estabelecimento de indicadores da atenção básica (Portaria GM $\left.\mathrm{n}^{\mathrm{o}} 3.925 / 98\right)$ e do início das atividades da Proposta de Avaliação de Desempenho do Sistema de Saúde (Proadess) (ALMEIDA, 2003). Paralelamente, no cenário internacional, capitaneada pela Organização Pan-Americana da Saúde, instituiu-se a Rede Interagência de Informações para a Saúde (Ripsa). Apresenta-se uma síntese temporal de iniciativas na figura 1. 
Figura 1. Experiências avaliativas e iniciativas de institucionalização das ações e práticas de monitoramento e avaliação no setor público da saúde

$1988 \quad$ Constituição Federal CF/88

1989

$1990 \quad$ |Lei no 8.080/90

1991

\begin{tabular}{l|l}
1992 & $\begin{array}{l}\text { Criação do Departamento de Controle, } \\
\text { Avaliação e Auditoria (DECA) }\end{array}$ \\
\hline
\end{tabular}

1993

$1994 \quad$ Ripsa

1995

1996

\begin{tabular}{l|l}
1997 & PPNASH \\
1998 & $\begin{array}{l}\text { Indicadores de Atenção Básica } \\
\text { PNASS }\end{array}$
\end{tabular}

PNASS $\quad$ Criação dos Departamentos:

$1999 \quad \begin{aligned} & \text { Nacional de Auditoria do SUS (Denasus) e } \\ & \text { de Controle e Avaliação de Sistemas (Decas) }\end{aligned}$

$2000 \quad$ World Health Report 2000 (WHR)

2001

Criação da Coordenação de Acompanhamento, Controle e Avaliação do Departamento de

$2002 \quad$ Proadess

Atenção Básica.

2003

2004

Transformação do Decas no

Departamento de Regulação,

Avaliação e Controle de Sistemas

(Derac)

2005

2006

Criação do Departamento Monitoramento

e Avaliação da Gestão do SUS

2007

(Demags)

2008

2009

\begin{tabular}{l|l}
2010 & $\begin{array}{l}\text { e-Car } \\
\text { IDSUS }\end{array}$ \\
\hline 2011 & $\begin{array}{l}\text { Criação do Departamento de } \\
\text { Monitoramento e Avaliação do SUS } \\
\text { (Demas) }\end{array}$
\end{tabular}

2012

2013 
Internamente, o Ministério da Saúde (MS) caminhou, nesse mesmo período, para a criação de estruturas administrativas voltadas às ações de monitoramento e avaliação do SUS. Em 1993, foi constituído e implantado o Departamento de Controle, Avaliação e Auditoria, que, em 2000, se desdobrou no Departamento Nacional de Auditoria do SUS (Denasus) e no Departamento de Controle e Avaliação de Sistemas (Decas). Nesse mesmo ano, foi criada a Coordenação de Informação no Departamento de Atenção Básica. Em 2003, oDecas foisucedidopelo Departamento de Regulação, Avaliação e Controle de Sistemas (Drac), quando também foi criada a Coordenação de Acompanhamento e Avaliação do Departamento Atenção Básica da Secretaria de Atenção à Saúde, substituindo a antiga Coordenação de Informação do Departamento de Atenção Básica. Em 2006, foi criado o Departamento de Monitoramento e Avaliação da Gestão do SUS (Demags), vinculado à Secretaria de Gestão Estratégica e Participativa.

Todos esses departamentos, componentes da estrutura do MS, tinham dentre suas principais funções as de monitoramento e avaliação. Em 2011, por meio do Decreto ${ }^{0}$ $7.530 / 11$, tais competências foram transferidas para o Departamento de Monitoramento e Avaliação do SUS (Demas) da Secretaria Executiva (SE).

O Demas surgiu para compatibilizar os instrumentos de gestão, criando norteadores institucionais para fundamentar a visão de futuro do MS, iniciativa inovadora de alteração do cenário pluralizado de conceitos, metodologias e instrumentos de M\&A existentes. Simultaneamente, tinha como foco, de forma partilhada com a Subsecretaria de Planejamento e Orçamento (SPO), implantar a integração entre Planejamento Estratégico e M\&A para modificação das práticas de gestão.

Dentre suas competências, cabe ao Demas, especialmente à Coordenação de Monitoramento e Avaliação, induzir e coordenar a formulação da Política de Monitoramento e Avaliação. A conformação de uma Política Nacional de Monitoramento e Avaliação para o Sistema Único de Saúde (PM\&A-SUS) tem, dentre seus objetivos, o de apresentar um paradigma para gestão do Sistema, diante da necessidade de sistematização e regulamentação específica em monitoramento e avaliação para o Setor.

Nesse sentido este artigo objetiva um resgate reflexivo do processo em contexto. O desafio proposto consiste em teorizar sobre uma estratégia ainda em construção, possibilitando compartilhar lições preliminares aprendidas à luz da gestão e permitir o debate junto à academia, organizações do setor saúde e da sociedade civil sobre a institucionalização das práticas de monitoramento e avaliação no âmbito do SUS.

\section{Metodologia}

O estudo do processo de formulação compreendeu a revisão bibliográfica sobre os processos de formulação e sobre a construção do marco institucional para monitoramento e avaliação no País nas bases PubmedMedline, Scientific Electronic Library Online (SciELO) e Centro Latino-Americano e do Caribe de Informação em Ciências da Saúde - Biblioteca Virtual em Saúde (Bireme-BVS); e a consulta descritiva e analítica dos documentos existentes, oriundos das reuniões do Grupo de Trabalho para a Elaboração da Política de Monitoramento e Avaliação do Sistema Único de Saúde (GT-PM\&A).

O conjunto de documentos analisados incluiu a legislação estruturante, os registros institucionais do MS, as atas de reuniões do GT-PM\&A, as diferentes minutas da PM\&A-SUS com o objetivo de apreender o processo de formulação, levando em consideração as três dimensões preconizadas por Champagne et al. (2011).

Para esses autores, a Apreciação Estratégica ou Análise Estratégica (AE) 
compreende a apreciação sobre a escolha já implantada ou em implementação. Assim, a AE objetiva compreender, ex post, a escolha e implantação de uma estratégia, a pertinência e a coerência dos fatores determinantes para o sucesso da intervenção escolhida, na realidade contextual de sua implementação. A pertinência e a coerência são fruto da interação que resulta em dada escolha, considerados os contextos técnico, socioeconômico, cultural e político-administrativo, os atores envolvidos e seus respectivos objetivos e interesses (SOARES, 2015).

Para cumprir suas atribuições de julgamento da pertinência, a $\mathrm{AE}$ foca em três componentes principais. O primeiro componente consiste na análise sobre adequação do processo de escolha do problema, quando se observa que o problema selecionado é de fato o foco da intervenção proposta. No caso em estudo, procurou-se rastrear os processos de produção das possíveis funções esperadas para a política em elaboração. Posteriormente, procede-se à análise da escolha dos objetivos da intervenção estudada e as soluções propostas, nesse caso, a formulação da PM\&A-SUS, ou seja, a pertinência e adequação das táticas propostas para a implementação da função previamente pactuada. O terceiro e último componente é a análise da pertinência das parcerias estratégicas mobilizadas, ou seja, diante da intervenção proposta, deve-se verificar o alinhamento das parcerias selecionadas para o enfrentamento do problema e questioná-lo frente ao contexto em que se desenvolve a intervenção (CHAMPAGNE ET AL., 2011).

A análise documental foi realizada utilizando-se a análise de conteúdo temática (BARDIN, 1977; SPINK, 2004). Procedeu-se à análise temática recortando-se, nas portarias, núcleos temáticos significantes associados às categorias da apreciação estratégica sua pertinência e adequação, contemplando-se os três componentes previamente descritos. Considerando-se que os propósitos inerentes a processos de monitoramento e avaliação se referem à regulação, responsabilização e produção de conhecimento, eles foram incorporados como organizadores dos temas selecionados, além, evidentemente, daqueles referentes às parcerias e escolhas alternativas.

\section{Resultados e discussão}

Pode-se dizer que o esforço de conformação de uma política de M\&A para o sistema de saúde nacional concretiza-se em um quadro de controvérsias, conflitos e consensos (FREY, 2000). Os resultados apresentados descrevem e analisam as portarias e as minutas, respeitando-se seu ordenamento cronológico e a proposta metodológica para a análise temática.

\section{O Decreto no 7.530/2011 e a Portaria no 1.517/24/07/2013: competências e o processo de formulação}

Em 2011, a gestão do MS deparou-se com a necessidade de harmonização de diversos instrumentos de gestão. Naquela época, estavam em vigor a fase final do Plano Plurianual (PPA 2008-2011), o Plano Mais Saúde, plano estratégico advindo da gestão anterior, o Plano Nacional de Saúde (PNS 2008-2011) e a Programação Anual de Saúde. Sim, ultimamente se iniciavam - até então correlacionadas, mas não propriamente integradas - as ações do PPA 2012-2015, do PNS 2012-2015 e do novo recorte estratégico do MS (BRASIL, 2015).

Diante disso e da complexidade do setor saúde, oportunizou-se a formação do Demas no âmbito da Secretaria Executiva do MS. Esse Departamento surgiu com a função de compatibilizar os instrumentos de gestão do setor saúde no intuito de criar norteadores institucionais. Assim, por meio do Decreto $\mathrm{n}^{\mathrm{o}}$ 7.530/2011, foi criado o Demas, subdividido em Coordenação-Geral de Monitoramento e Avaliação (CGMA) e Coordenação-Geral de 
Gestão da Informação Estratégica (CGGIE). Compete ao Demas (i) coordenar a formulação da Política de Monitoramento e Avaliação (PMA-SUS); (ii) coordenar os processos de elaboração, negociação, implantação de normas, instrumentos e métodos necessários ao fortalecimento das práticas de monitoramento e avaliação do SUS; (iii) articular e integrar as ações de monitoramento e avaliação executadas pelos órgãos e unidades do MS; (iv) desenvolver metodologias e apoiar iniciativas que qualifiquem o processo de monitoramento e avaliação do SUS; (v) viabilizar e coordenar a realização de estudos e pesquisas visando à produção do conhecimento no campo do monitoramento e avaliação do SUS; (vi) participar da coordenação do processo colegiado de monitoramento, avaliação e gestão das informações do SUS; e (vii) sistematizar e disseminar informações estratégicas para subsidiar a tomada de decisão na gestão federal do SUS (BRASIL, 2015).

As atividades do Demas, nos primeiros anos após sua criação, concentraram-se nas macro dimensões técnico-administrativas de (i) construção, coordenação e monitoramento do planejamento estratégico do MS; (ii) reestruturação da Sala de Apoio à Gestão Estratégica (Sage); e (iii) coordenação na formulação da PM\&A-SUS.

A Portaria $n^{0} 1.517$, de 24 de julho de 2013, inicia, institucionalmente, o processo de formulação da PM\&A-SUS por instituir o Grupo de Trabalho para a Elaboração da Política de Monitoramento e Avaliação do Sistema Único de Saúde (GT-PM\&A-SUS).

O GT-PM\&A-SUS é composto por um representante do MS, Fundação Oswaldo Cruz (Fiocruz), Conselho Nacional de Secretários de Saúde (Conass) e Conselho Nacional de Secretarias Municipais de Saúde (Conasems), sendo que o MS tem o direito de expandir sua representação a até sete membros por meio da incorporação de até um representante de cada secretaria finalística do órgão.
A análise documental da Portaria que instituiu o grupo de trabalho para formulação da PM\&A-SUS permitiu apreender um processo de construção das prerrogativas, que retroagem às legislações que regem $o$ SUS. Pela legislação, a responsabilidade na definição de instâncias e mecanismo para avaliação das ações e serviços de saúde é dos entes federados, tendo a União, por meio do MS, a prerrogativa legal de monitoramento, avaliação e disseminação das ações e serviços de saúde.

Apesar de ser tácita e explícita a compreensão dos gestores da utilidade do M\&A como ferramenta de gestão e tomada de decisão, bem como das várias iniciativas anteriores de setores do MS, as ações que inauguram a formalização de uma política nacional de M\&A, paradoxalmente, só ocorrem após 25 anos após o estabelecimento da responsabilidade do MS no processo. Como descrito no quadro 1, a Portaria ${ }^{\circ} 1.517$, de 24 de julho de 2013, cujo caput propõe a elaboração da PM\&A-SUS, fomenta a expectativa da formação de um sistema nacional de avaliação em saúde.

O processo de formulação de uma política exige um planejamento além do conjunto de iniciativas que deve, a princípio, envolver democraticamente o conjunto de interessados (LINDBLOM, 1981 APUD PAESE; AGUIAR, 2012). Nesse sentido, um aspecto importante é notar que é rotina das ações no MS, na constituição de grupos de trabalho, prever um representante de todas as secretarias como pressuposto de transversalidade das ações, o que raramente ocorre de fato.

Além disso, a análise do texto sugere a concentração da representação acadêmica em uma instituição, posição revista pelo grupo de trabalho e pelo convite a outras instituições para a participação de forma complementar. Por fim, observa-se a ausência do controle social no texto, onde o processo preliminar de formulação inclui apenas a gestão e a academia. 
Quadro 1. A elaboração da PM\&A-SUS: fatos e versões

Documento

Portaria no 1.517 , de 24 de julho de 2013.

Minuta PMA-SUS, de 4 de dezembro de 2013.

Súmula da 1 a reunião do Grupo de Trabalho para a Elaboração da Política de Monitoramento e Avaliação do Sistema Único de Saúde, em 09 de dezembro de 2013.

Minuta PMA-SUS, de 9 de dezembro de 2013.

Minuta PMA-SUS, de 10 de dezembro de 2013.

Minuta PMA-SUS, de 07 de abril de 2014
Tipologia

Legislação relacionada

Minuta da Política

Documentos relacionados

Minuta de portaria

Minuta de portaria

Minuta de portaria

Minuta de portaria

\section{Pertinência do problema} focalizado

Parte da prerrogativa instituída no ordenamento jurídico fundados do SUS, cuja responsabilidade na definição de instâncias e mecanismo para avaliação das ações e serviços de saúde é dos entes federados.

Aborda o problema a partir da visão institucional do MS.

Iniciam-se questionamentos sobre a necessidade de existência de um PMA para além da gestão.

Apresenta pressupostos para a conceituação e melhor definição do problema a ser enfrentado.
Pertinência dos objetivo

Intenta a elaboração de uma proposta da PMA-SUS como produto imediato e consequentemente um insumo à construção de um sistema de avaliativo do SUS.

Apresenta grande marcos na perspectiva da accountability.

Iniciam-se as preocupações com o marco teórico que guiará a política; aparecem os primeiros questionamentos sobre os objetivos e dimensões da PMA-SUS.

Traz o pressuposto da atribuição de mérito e valor por meio das práticas avaliativas e a influência do contexto sob o processo avaliativo. Questiona a ausência de preocupações quanto à institucionalização do M\&A e ao desenvolvimento do conhecimento no campo do $M \& A$

Não evidencia alterações nesta dimensão.

blema em contexto; explicit iniciativas e experiências de monitoramento e avaliação de M\&A já desenvolvidas no âmbito do SUS.

Aprofunda o contexto apresentando iniciativas e experiências ainda não abordadas no texto anterior, nacionais e internacionais; aborda aspectos tangentes à temática do planejamento.

Incorpora as discussões anteriores na definição do problema.

incorporando aspectos do fomento ao conhecimento, às estratégias em curso e ao desenvolvimento de novas iniciativas avaliativas; bem como auxílio ao monitoramento do PPA; inclui a avaliação de desempenho do SUS nas diretrizes da PMA-SUS.

Retoma a inclusão de a PMA-SUS ter abrangência sobre sistema e ações de saúde
Pertinência da parceria estratégica

Inclui-se um representante de cada secretaria do MS, a Fundação Oswaldo Cruz e as instâncias colegiadas de gestores (Conass e Conasems). Possibilita a participação de outros atores, especialistas no tema, de forma complementar.

Limitam-se às instâncias gestoras do SUS, nas três esferas de gestão.

Questiona-se a abrangência da PMA-SUS para além do setor público e a inclusão de usuários e prestadores de serviços no conjunto de atores envolvidos.

Evidencia o caráter colaborativo das ações avaliativas, entretanto não sugestiona a inclusão de parcerias.

Não evidencia alteracões nesta dimensão

Não evidencia alterações nesta dimensão. públicas e privadas.
Retoma a inclusão dos prestadores de serviço como partícipes da PMA-SUS;

Questiona a inclusão das fundações e entidades vinculadas ao MS 
O primeiro esboço da $\mathrm{PM} \& \mathrm{~A}$, de 4 de dezembro de 2013, data de cinco meses após a publicação da Portaria $\mathrm{n}^{0} 1.517 / 2013$. O texto traz diretrizes abrangentes e transversais às áreas técnicas do MS, como descrito a seguir.

\section{Diretrizes:}

VI. Subsídios aos processos de responsabilização, contratualização de metas, resultados e melhoria de desempenho de trabalhadores e gestores no âmbito do SUS;

VII. Fomento e contribuição para maior excelência, eficiência, eficácia e efetividade na produção de bens, ações e serviços públicos de saúde;

IX. Contribuição para integração das ações de monitoramento e avaliação das políticas públicas intersetoriais;

X. Articulação e estabelecimento de parcerias com órgãos governamentais e não governamentais nacionais e internacionais intra e intersetoriais, bem como com a sociedade civil organizada para o fortalecimento das ações de monitoramento e avaliação em saúde. (BRASIL, 2013, P. 8).

No documento, não se observa a explicitação das parcerias, tais como da academia e do controle social, nem no processo de formulação nem como protagonistas na implantação da política. Os atores corresponsáveis acima se limitam às instâncias gestoras nas três esferas do SUS. Apesar de o texto trazer a palavra 'participação social', a expressão aparece de maneira isolada e pouco integrada ao restante do conteúdo do material analisado.

O documento, ainda bastante incipiente, privilegia M\&A para a gestão federal do SUS. Isso pode ser apreendido pela análise do texto em que se explicitam as ações macro a serem desenvolvidas pelo governo federal, considerando-se os demais entes como seguidores.

A minuta faz referências frequentes a uma dada concepção de accountability. A crescente preocupação dos gestores com a responsabilização civil da improbidade administrativa parece transparecer no texto, limitando os propósitos e usos do monitoramento e da avaliação. Assim, o objetivo central da proposta em construção parece expressar mais as preocupações dos gestores públicos com as lentes dos órgãos de controle do que com as finalidades de uma política de M\&A.

Outro aspecto relevante, é que apenas os estados e municípios são explicitamente chamados a incluir a Educação Permanente em M\&A em seus territórios. Essa função não é mencionada no que se refere à gestão federal. Esse ponto suscitou algumas questões tais como: seria a União autossuficiente em capacidade avaliativa? Já possui conhecimento, atitudes e práticas em nível de maturidade satisfatório? Não precisa desenvolver mecanismos reflexivos posicionais referentes às funções federais?

Em nove de dezembro de 2013, tem-se a I Reunião do Grupo de Trabalho para a Elaboração da Política de Monitoramento e Avaliação do Sistema Único de Saúde. O grupo presente envolveu 21 representantes dos seguintes órgãos e entidades: MS (13), Fiocruz (2), Conasems (1), Rede Unida (1), Associação Brasileira de Saúde Coletiva (Abrasco) (2), Associação Brasileira de Economia da Saúde (ABrES) (1) e Centro Brasileiro de Estudos de Saúde (Cebes) (1).

Apreendeu-se da leitura da súmula dessa reunião algumas frentes de questionamento, preocupações e proposições dos participantes. Inicialmente, destacamse: (a) preocupações sobre a expansão do normativo da PM\&A-SUS além da gestão e do setor público, que incluiria as demais esferas de gestão e o setor privado também elementos do SUS; (b) questionamentos sobre as dimensões de avaliação que a 
PMA-SUS pretende atingir; (c) importância e necessidade de se definirem os objetivos de forma clara, participativa e integrada, e utilizando-se das experiências anteriores; (d) questionamento quanto à função da PMASUS. Uma questão presente era se o texto normativo prestar-se-ia a definir conceitos, terminologias e técnicas padronizadas, seja para a gestão do monitoramento e avaliação seja para o monitoramento e avaliação para gestão. Outro ponto enfatizado foram as possibilidades de avaliação no âmbito do SUS; e, por fim, emergem os primeiros questionamentos sobre o uso exclusivo do M\&A para accountability, discutindo-se que 'o aspecto reflexivo pedagógico deve prevalecer na avaliação', em contraste ao propósito da auditagem (sic, participante da reunião).

Observa-se, no resumo da fala dos participantes, a multiplicidade de ações de avaliação desenvolvidas no âmbito do MS com diferentes noções, conceitos e técnicas que prevalecem na penumbra de um processo sistematizado e organizado de M\&A na instituição, tendo a rotatividade de gestores uma parcela considerável de influência nesse aspecto.

Dando-se continuidade às ações, a minuta de nove de dezembro do mesmo ano, produzida logo após a primeira reunião presencial do GT, foi submetida a um leitor crítico, especialista em M\&A. A apreciação realizada reforça os diferentes propósitos do M\&A que uma política deve considerar, sejam eles a verificação de conformidade muito própria do monitoramento, a imputabilidade, a apreciação de mérito ou valor e a produção de conhecimento. A leitura crítica traz a concepção de que o processo avaliativo permite a tradução e mobilização de conhecimento com intensa negociação de valores e interesses, a mediação de diferentes expectativas e mesmo de processos emancipatórios, inclusive de diferentes concepções de bem estar social. Outro aspecto relevante é o alerta para a inexistência de processos que favoreçam o fomento ao conhecimento em M\&A e a consequente proposição para a inclusão de aspectos relativos ao incentivo à pesquisa, desenvolvimento e inovação no campo do M\&A no texto normativo então em construção.

No dia posterior, a minuta com as contribuições de revisor especializado é revista pelo Coordenador-Geral de Monitoramento e Avaliação do MS. Ele se aprofunda na conceituação do problema, em especial no dissertar sobre o contexto em que este está envolvido o M\&A no âmbito do MS; e explicita iniciativas e experiências de monitoramento e avaliação já desenvolvidas no âmbito do SUS, assim reconhecendo que há um percurso já trilhado de M\&A do SUS, porém é perceptível a ênfase no Programa Nacional de Avaliação de Serviços Hospitalares (PNASH), Programa Nacional de Avaliação dos Serviços de Saúde (PNASS), Índice de Desempenho do SUS (IDSUS) e Índice de Desempenho da Saúde Suplementar (IDSS), todas estas inciativas diretas do referido coordenador, seja na função que ocupara à época ou em trajetória anterior de atuação no sistema público de saúde do País.

Após um período de aproximadamente quatro meses destinado ao recebimento das contribuições oriundas dos participantes da primeira reunião do GT-PM\&A-SUS, tem-se a minuta de sete de abril de 2014. Essa quarta versão traz um conjunto importante de questionamentos, visões e proposições dos atores envolvidos no processo e participantes do momento presencial anterior.

Retoma-se o olhar para o monitoramento como instrumento de acompanhamento de metas, renascendo, consequentemente, a forte tendência do reducionismo dos propósitos do monitoramento e avaliação e suas implicações para a elaboração do documento sobre a política. Nas sugestões, observa-se um desvio da discussão da função da política para a problematização do M\&A como instrumento de acompanhamento do desempenho de ações planejadas, incluindo-se no 
texto, dentre os objetivos da PMA-SUS, o de se desenvolver e implantar um instrumento para subsidiar o acompanhamento dos componentes da saúde no PPA.

O documento deixa claro que 'o M\&A são elementos estratégicos para o Planejamento', subentendendo-se o papel do M\&A como elemento de regulação, controle e responsabilização. A tomada de decisão baseada em evidências, termo frequente nos documentos atuais de gestão pública, parece ser uma novidade, entretanto, já nos anos 1930, defendia-se a possibilidade de conciliar o conhecimento acadêmico com a atuação empírica dos governos, além de se propagar ideias sobre a capacidade de diálogo entre a academia, gestores e os demais grupos de interesse. Mais tarde, em 1960, argumentava-se a capacidade de racionalizar a ação dos gestores por meio da institucionalização de estruturas capazes de modelar o comportamento dos decisores na busca pelos resultados pretendidos e, por fim, veio à tona a defesa de uma perspectiva mais dinâmica sobre a tomada de decisão como a influência do contexto político, institucional e social. Racionalidade técnica não é fator isolado, único, nem o principal para a tomada de decisão (PAESE; AGUIAR, 2012).

A análise do documento da minuta de sete de abril evidencia a ampliação dos objetivos, incorporando aspectos do fomento ao conhecimento tanto das estratégias em curso como do desenvolvimento de novas iniciativas avaliativas, além da inclusão da avaliação de desempenho do SUS como diretriz da PM\&A-SUS. O documento detém-se a incrementar o contexto do M\&A no SUS, por meio da incorporação de iniciativas e experiências ainda não abordadas no texto normativo anterior, inclusive das experiências internacionais.

Apesar de expandir as experiências avaliativas, limita-se às ações desenvolvidas no âmbito da gestão federal do Sistema e privilegia a experiência do Proadess. O Proadess consistiu-se em iniciativa desenvolvida em parceria entre a Fiocruz e a Abrasco, em atendimento ao 'World Health Report' publicado pela Organização Mundial da Saúde nos anos 2000, que propôs metodologia de avaliação para o sistema brasileiro de saúde. Constituiu-se em metodologia materializada de modelo para avaliação de desempenho de sistemas de saúde (ALMEIDA, 2003).

A quinta versão, de treze de maio de 2014, contempla alterações morfossintáticas e gramaticais, sem inclusão ou modificação relevante do conteúdo. No mesmo dia, na sexta e última versão disponível, são incorporadas as considerações do Conass, que substancialmente retomam as proposições de que a PM\&A-SUS a) tenha abrangência sobre sistema e ações de saúde públicas e privadas; b) inclua os prestadores de serviço como partícipes do processo de elaboração, implementação e execução da política; e c) inclua as fundações e entidades vinculadas ao MS que compõem o SUS como protagonistas do processo avaliativo.

Pôde-se apreender que as considerações do Conass apresentam as funções esperadas do gestor do sistema e albergam reflexões além da estrutura do MS e dos serviços de saúde públicos em seu stricto sensu, evidenciando a relação necessária e indissociável do setor saúde com os prestadores de serviços privados e filantrópicos, unidades vinculadas e fundações de pesquisa em saúde que compõem o sistema. Entretanto, as minutas propostas não dialogam com outras representações, como as de movimentos sociais que não se encontrem representadas nos Conselhos.

\section{Lições aprendidas}

As políticas públicas expressam a intenção de um governo em exercer a transformação sobre determinado problema ou interesse da sociedade. Nesse sentido, a ausência da ação deve também deve ser abordada como escolha de um governo, uma vez que 
se configura como forma de manifestação política e de postura do Estado.

Os processos de formulação de políticas no setor público raramente são frutos exclusivos da aplicação de métodos e técnicas de planejamento. Na realidade, são conformados e expressam interesses e coalizões de grupos hegemônicos em uma dada conjuntura política. Dessa forma, o processo de formulação, que parece ser conduzido pelo 'entusiasmo' pessoal do gestor ou de especialistas, explicita controvérsias ao materializar influências positivas, antagonismos e resistências contrárias, intra e interinstitucionais.

Além disso, é tácita e de longa data a preocupação dos gestores com a implantação de meios capazes de responder ao monitoramento e avaliação das ações e serviços públicos, em especial no setor saúde, seja por meio da estruturação hierárquica administrativa ou pelo incentivo às iniciativas avaliativas em parceria com instituições de ensino e pesquisa.

No contexto da PM\&A-SUS, o processo de indução deu-se na perspectiva de formulação da política como norteadora da institucionalização do monitoramento e avaliação na saúde, com pouca consideração pela conjuntura política vigente e destacável desconsideração a processos de inovação. $\mathrm{Na}$ linguagem coloquial poderíamos dizer que houve 'mais do mesmo'.

O caráter dinâmico e plural do setor saúde constrói, frequentemente, barreiras à formulação de políticas. A existência de uma política condicionada à existência de consensos, mesmo que provisórios, é antagonizada por forças de toda ordem, sejam questões concretas, como rotatividade de gestores com consequente reorganização dos arranjos de governança, sejam questões orçamentárias e mais recentemente associadas à judicialização, que tendem a dificultar e em, alguns casos, paralisar os processos até então desenvolvidos.

A construção de um documento normativo que contivesse os princípios e diretrizes, tomado como estratégia para a formulação da política de M\&A para o SUS, pode ser considerada sob dois pontos hipotéticos a serem explorados na continuidade deste estudo. O primeiro presume que o processo de formulação do documento acomode e materialize os diferentes interesses dos atores envolvidos no processo de formulação da política. Ou seja, a formulação do documento viabiliza a construção de alianças, configurando-se como um inscrito orgânico de grupos hegemônicos. O segundo tende a exprimir a lógica inversa, isto é, em sistemas políticos, de hegemonia notadamente republicana, a convivência de diversos e divergentes interesses acomoda arranjos políticos orgânicos dinâmicos. A existência do inscrito é consequência e não causa da organicidade. A escolha da estratégia e ela própria são, assim, balizadas pelo contexto político e a ele respondem.

A descontinuidade dos encontros do GT, as constantes alterações em diversos cargos de gestão diretamente envolvidos com o processo de formulação, a dificuldade de alinhamento de interesses e, em especial, a concepção predominante de monitoramento e avaliação como instrumento de avaliação da gestão por resultados, ligadas à judicialização crescente dos processos de gestão, já prenunciavam a instabilidade do contexto político pouco presente na formulação do documento da PNII. Este último ponto, especialmente o caráter valorativo indiscutível conferido a alcance de metas e efeitos particulares e capaz de cristalizar situações particulares em normas e leis, contribuiria para corromper a noção de 'padrões', enrijecendo e dificultando sobremaneira a possibilidade dos necessários arranjos contextuais tático-estratégicos (GOODIN, 1984). Dessa forma, considera-se necessário para continuidade do estudo uma análise detalhada dos atores envolvidos, incluída em estudo exploratório de conjuntura. 


\section{Referências}

\author{
ALMEIDA, C. Relatório final do projeto \\ desenvolvimento de metodologia de avaliação do \\ desempenho do sistema de saúde brasileiro. Rio de \\ Janeiro: Proadess, 2003. Disponível em: <http:// \\ proadess.icict.fiocruz.br/relatoriofinal.pdf $>$. Acesso \\ em: 10 fev. 2016
}

BARDIN, L. Análise de Conteúdo. Lisboa: Edições 70, 1977.

BRASIL. Decreto n ${ }^{\circ} 7.530$, de 21 de julho de 2011. Aprova a Estrutura Regimental e o Quadro Demonstrativo dos Cargos em Comissão e das Funções Gratificadas do Ministério da Saúde. Diário Oficial [da] União, Brasília, DF, 21 jul. 2011. Disponível em: <http:// www.planalto.gov.br/ccivil_03/_Ato2011-2014/2011/ Decreto/D7530.htm>. Acesso em: 10 fev. 2016.

Lei ${ }^{0}$ 8.080, de 19 de setembro de 1990. Dispõe sobre as condições para a promoção, proteção e recuperação da saúde, a organização e o funcionamento dos serviços correspondentes e dá outras providências. Diário Oficial [da] União, Brasília, DF, 19 set. 1990. Disponível em: <http://www.planalto.gov.br/ccivil_03/ leis/L8080.htm>. Acesso em: 2 fev. 2016.

Ministério da Saúde. Minuta da Política Nacional de Monitoramento e Avaliação do SUS, Grupo de Trabalho para a Elaboração da Política de Monitoramento e Avaliação do Sistema Único de Saúde (SUS). Departamento de Monitoramento e Avaliação do SUS (Demas), Brasília, DF, 4 dez, 2013. Mimeo.

Ministério da Saúde. Departamento de Monitoramento e Avaliação do SUS. Planejamento estratégico do Ministério da Saúde 2011-2015: resultados e perspectivas. 5. ed. Brasília, DF: Ministério da Saúde, 2015. Disponível em: <http://bvsms.saude.gov.br/bvs/ publicacoes/planejamento_estrategico_ministerio_ saude_2011_2015_5ed.pdf>. Acesso em: 10 fev. 2017.

Ministério da Saúde. Portaria no 3.408, de 5 de agosto de 1998. Institui o programa nacional de avaliação dos serviços hospitalares. (ementa elaborada pela CDI/MS). Diário Oficial [da] União, Brasília, DF,
05 ago. 1998. Disponível em: <http://www.husm.ufsm. br/janela/legislacoes/hemato-oncologia/centrosoncologia/portaria-no-3409gm-de-05-de-agostode-1998.pdf>. Acesso em: 2 fev. 2016.

Ministério da Saúde. Portaria no 3.928, de 13 de novembro de 1998. Aprova o Manual para Organização da Atenção Básica no Sistema Único de Saúde, constante do Anexo I desta portaria. Diário Oficial [da] União, Brasília, DF, 17 nov., 1998. Disponível em: <http://bvsms.saude.gov.br/bvs/saudelegis/gm/1998/ prt3925_13_11_1998_rep.html>. Acesso em: 2 fev. 2016.

Ministério da Saúde. Portaria no ${ }^{\circ}$.517, de 24 de julho de 2013. Institui o Grupo de Trabalho para a Elaboração da Política de Monitoramento e Avaliação do Sistema Único de Saúde (SUS). Diário Oficial [da] União, Brasília, DF, 24 jul. 2013. Disponível em: <http://bvsms.saude.gov.br/bvs/saudelegis/gm/2013/ prt1517_24_07_2013.html>. Acesso em: 11 fev. 2016.

CHAMPAGNE, F. et al. A Análise Estratégica. In: BROUSSELlE, A. et al. (Org.). Avaliação: conceitos e métodos. Rio de Janeiro, Fiocruz, 2011.

FREY, K. Políticas públicas: um debate conceitual e reflexões referentes à prática da análise de políticas públicas no Brasil. Planejamento e Políticas Públicas, Brasília, DF, v. 21, p. 211-259, 2000.

GOODIN, R. Political Theory and Public Policy. Chicago: University of Chicago Press, 1984.

JANNUZZI, P. M. Monitoramento e avaliação de programas sociais: uma introdução aos conceitos e técnicas. Campinas: Alínea, 2016.

LINDBLON, C. E. O processo de decisão política. Brasília, DF: UNB, 1981.

MACHADO, S. H. S. O uso da teoria de stakeholders em uma análise da etapa de formulação da Política Nacional de Medicamentos. Rev. Adm. Pública, Rio de Janeiro, v. 47, n. 3, p. 543-565, maio/jun. 2013. 
MATTOS, R. A.; BAPTISTA, T.W.F. (Org.). Caminhos para análise das políticas de saúde. Porto Alegre: Rede Unida, 2015.

NEIROTTI, N. Evaluation in Latin America: paradigms and practices. Evaluation voices from Latin America. New directions for evaluation, San Francisco, n. 134, p. 7-16, 2012.

PAESE, C. R.; AGUIAR, A. A. G. Revisitando os conceitos de formulação, implementação e avaliação de políticas e programas sociais no brasil. Revista NUPEM, Campo Mourão, v. 4, n. 6, p. 65-87, jan./jul. 2012.

SANTOS, E. M. A importância da avaliação no contexto das políticas de saúde no Brasil. 2013. Disponível em: $<$ http://www.extranet.ead.fiocruz.br/criacao/as/txts/ ual/modl/ual_mod1_importancia_avaliacao.pdf $>$. Acesso em: 19 fev. 2016.

SIDNEY, M. S. Policy Formulation: design and tools. In: FISCHER, F.; MILLER, G. J.; SIDNEY, M. S. Handbook of public policy analysis: theory, politics, and methods. Boca Raton: CRC Press, 2007, p. 79-87.

SOARES, B. C. Análise estratégica das ações de vigilância epidemiológica do Programa de Controle da Tuberculose no município de Itaboraí (RJ). 2015. 93 f. Dissertação (Mestrado Profissional em Saúde Pública) - Escola Nacional de Saúde Pública Sergio Arouca,
Fundação Oswaldo Cruz, Rio de Janeiro. 2015.

SOUZA, C. Políticas Públicas: uma revisão da literatura. Sociologias, Porto Alegre, v. 8, n. 16, p. 20-45, jul./dez., 2006.

SPINK, P. Análise de documentos de domínio público. In: SPINK. M. J. (Org.). Práticas discursivas e produção de sentidos no cotidiano: aproximações teóricas e metodológicas. São Paulo: Cortez, 2004. p. 123-151.

TEIXEIRA, E. C. O papel das políticas públicas no desenvolvimento local e na transformação da realidade. Salvador: AATR; 2002.

VIACAVA, F. et al. Uma metodologia de avaliação do desempenho do sistema de saúde brasileiro. Ciênc. saúde coletiva, Rio de Janeiro, v. 9, n. 3, p. 711-724, set. 2004.

VIANA, A. L. D.; BAPTISTA, T. W. F. Análise de Políticas de Saúde. In: GIOVANELLA, L. (Org.). Políticas e Sistemas de Saúde do Brasil. 2. ed. Rio de Janeiro: Fiocruz, p. 59-87, 2012.

\footnotetext{
Recebido para publicação em abril de 2016

Versão final em janeiro de 2017

Conflito de interesses: inexistente

Suporte financeiro: não houve
} 